Vietnam Journal of Mechanics, VAST, Vol.29, No.1 (2007), pp. 13-24

\title{
APPLICATION OF THE CURVATURE SMOOTHING TECHNIQUE FOR FOUR-NODE QUADRILATERAL REISSNER-MINDLIN PLATE ELEMENT
}

\author{
Nguyen Xuan Hung, Ngo Thanh Phong \\ Department of Mathematics and Informatics, \\ University of Natural Sciences - VNU-HCM
}

\begin{abstract}
A quadrilateral element with smoothed curvatures for Reissner-Mindlin structure plates is proposed. A curvature matrix at an arbitrary point is normalized by a non-local approximation over a smoothing function. By choosing a constant smoothed function and applying the divergence theorem, the bending stiffness matrix calculated on boundaries of smoothing elements (smoothing cells) instead of on their interior. Several numerical results are analyzed to demonstrate high reliability and free locking of the proposed method.
\end{abstract}

\section{INTRODUCTION}

The plate structure has been played a significant important role in the application to engineering analysis. The study of its behaviour has been for long time and the plate bending in which it contains complex problems still continuous to be discussed by engineers and scientists. In the theory of plates there are two different cases of plates that are the Kirchhoff and the Reissner - Mindlin plate. In the intuitive answer, engineers normally will choose the Reissner - Mindlin plate as being to use the conforming $C^{0}$ - elements for approximations. An advantage of the Reissner-Mindlin model over the biharmonic plate model is that the energy involves only first derivatives of the unknowns and so conforming finite element approximations require the use of $C^{0}$ - class element instead of the required $C^{1}$ - element for the biharmonic model. As shown in the literatures, the numerical analysis of the Reissner - Mindlin theory has to use a special care in order to avoid the so-called shear locking phenomenon due to the limit of the small thickness. The development of general procedures to overcome this drawback is an active research area. As the thickness tends to zero, the Krichhoff constraint appears in the Reissner - Mindlin model and locking phenomenon is frequently met if low-order elements are employed. There are many ways to overcome shear locking phenomenon and stability such as a reduced integration or a selective reduced integration, see Reference [1], on other terms of the strain energy. For an example with a four-node quadrilateral element, the $2 \times 2$ Gauss point integration is considered for the bending strain energy and the $1 \times 1$ Gauss point integration is used for the shear strain energy. Concerning on this research topic, a lot of publications has found in the literatures $[2,3]$.

Considering a general quadrilateral plate element, a start of work is to interpolate the deflection and the two rotations of four-nodal element. An approach used collocation constrain of shear approximation on boundary element then was proposed by Bathe et al. [5] for bilinear plate element. Here the discretized fields consist of the later displacement, 
the rotations and shear strain. While the deflection and the rotations continuous employing $C^{0}$ - approximation, the shear strain is interpolated by points on the edges of element. This method give a good result and free locking. It is known as MITC4 or Bathe-Dvorkin element [5]. Many versions then have been developing this method for the high degree of approximate fields, see e.g the textbook [3].

To resolve shear locking in mesh-free methods for the Reissner - Mindlin model, a stabilized conforming nodal integration (SCNI) has been proposed as a normalization for nodal integration $[8,9]$ of meshfree Galerkin weak form. In this approach, the strain smoothing stabilization has been introduced in SCNI to meet integration constraints and thus fulfills the linear exactness in the Galerkin approximation of the second order partial differential equations. Then Wang and Chen (2004) [4] also have shown that the cause of shear locking in Mindlin - Reissner plate formulation is due to inability in the approximation functions to reproduce Kirchhoff mode, and the incapability of the numerical method to achieve pure bending exactness $(\mathrm{BE})$ in the Galerkin approximation. In this paper, a curvature smoothing method (CSM) is proposed to normalize strain fields.

In the mesh-free methods using stabilized nodal integration, the entire domain is discretized into cells defined by the field nodes, such as the cells of a Voronoi diagram $[8,9]$. Integration is performed along the edges of each cell. Although meshfree methods obtain good accuracy and high convergence rate, the non - polynomial or usually complex approximation space increases the computational cost of numerical integration. Recently, applications of the SCNI to the FEM had been developed by authors such as Liu et al. [6], Nguyen et al. [7] for 2D problems. It shown that the FEM with smoothed techniques gives stabilized results and high accuracy.

The aim of this paper is to extend the application of the SCNI to the FEM for plates. A curvature smoothing technique is utilized to compute the bending strains. The shear strains is approximated by an independent interpolation fields in the natural co - ordinate system. A new element that overcomes the shear locking is proposed.

The outline of the paper is organized as follows. In the next section we present the basic equations of plate problem and weak form. The curvature smoothing stabilization and the finite element discretization using the CSM are introduced in section 3. Several numerical examples are given in Section 4. Finally, Section 5 closes some conclusions and further works.

\section{GOVERNING EQUATIONS AND WEAK FORM}

Let $\Omega$ be the region in $R^{2}$ occupied by the middle plane of the plate. $w$ and $\beta=$ $\left(\beta_{x}, \beta_{y}\right)^{T}$ denote the transverse displacement and the rotations in the $\mathrm{x}-\mathrm{z}$ and $\mathrm{y}-\mathrm{z}$ planes, see Fig. 1, respectively. For simplicity, we will consider problems with the hard clamped boundary conditions. However, it can do well for several other boundary conditions. Assuming that the material is homogeneous and isotropic with Young's modulus $E$ and Poisson ratio $z$, the governing differential equations of Reissner-Mindlin plate are of the form,

$$
\begin{gathered}
-\operatorname{div} C^{b} \kappa(\boldsymbol{\beta})-\lambda \operatorname{t\gamma }(\boldsymbol{\beta})=0 \text { in } \Omega, \\
-\lambda \operatorname{tdiv}(\boldsymbol{\gamma})=p \text { in } \Omega, \\
w=\bar{w}, \boldsymbol{\beta}=\overline{\boldsymbol{\beta}} \text { on } \Gamma=\partial \Omega,
\end{gathered}
$$


where $t$ is the plate thickness, $p=p(x, y)$ is the transverse loading per unit area, $\lambda=$ $\frac{k E}{2(1+\nu)}, k=\frac{5}{6}$ is the shear correction factor and $\mathbf{C}^{b}$ is the tensor of bending moduli, $\kappa$ and $\gamma$ are the bending and shear strains, respectively, defined by

$$
\kappa=\left[\begin{array}{c}
\frac{\partial \beta_{x}}{\partial x} \\
-\frac{\partial \beta_{y}}{\partial y_{\partial}} \\
\frac{\partial \beta_{x}}{\partial y}-\frac{\partial \beta_{y}}{\partial x}
\end{array}\right], \gamma=\left[\begin{array}{c}
\frac{\partial w}{\partial x}+\beta_{x} \\
\frac{\partial w}{\partial y}-\beta_{y}
\end{array}\right] .
$$

The Equations $(2.1)-(2.3)$ correspond to the minimization of the total potential function

$$
\Pi=\frac{1}{2} \int_{\Omega} \mathbf{C}^{b}: \kappa: \kappa d \Omega+\frac{1}{2} \int_{\Omega} \mathbf{C}^{s}: \gamma: \gamma d \Omega-\int_{\Omega} w p d \Omega .
$$

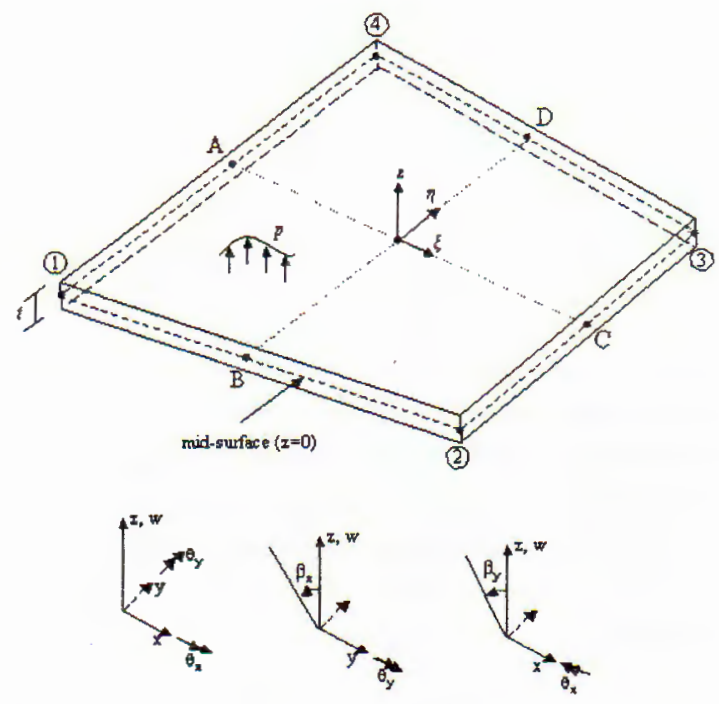

Fig. 1. Assumption of shear deformations for quadrilateral plate element

Assumed that the bounded domain $\Omega$ is discretized into $n_{e}$ finite elements, $\Omega \approx \Omega^{h}=$ $\sum_{e=1}^{n_{e}} \Omega^{e}$. The finite element solution $\mathbf{u}^{h}=\left[\begin{array}{lll}w & \beta_{x} & \beta_{y}\end{array}\right]^{T}$ of a displacement model for ReissnerMindlin plate is expressed as follows

$$
\mathbf{u}^{h}=\sum_{i=1}^{n p}\left[\begin{array}{ccc}
N_{i} & 0 & 0 \\
0 & 0 & N_{i} \\
0 & N_{i} & 0
\end{array}\right] \mathbf{q}_{i},
$$

where $n p$ is total the number of element nodes, the $N_{i}$ 's are the bilinear shape interpolation functions associated to node $i$, the $\mathbf{q}_{i}=\left[w_{i} \theta_{x i} \theta_{y i}\right]^{T}$ are the nodal degrees of freedom of the variables $\mathbf{u}^{h}=\left[\begin{array}{lll}w & \beta_{x} & \beta_{y}\end{array}\right]^{T}$ associated to node $i$. Then, the discrete curvature field is

$$
\kappa^{h}=\mathbf{B}^{b} \mathbf{q}
$$


where $\mathbf{B}^{b}$ defined below is the curvature to nodal displacement matrix. The approximation of shear strain is written by

$$
\gamma^{h}=\mathbf{B}^{s} \mathbf{q}
$$

where

$$
\mathbf{B}_{i}^{s}=\left[\begin{array}{ccc}
N_{i, x} & 0 & N_{i} \\
N_{i, y} & -N_{i} & 0
\end{array}\right] .
$$

By substituting Eq. (2.6) - Eq. (2.8) into Eq. (2.5) and minimization, we obtain a linear system of an individual element for the vector of nodal unknowns q,

$$
\mathrm{Kq}=\mathbf{g},
$$

with the element stiffness matrix given by

$$
\mathbf{K}=\int_{\Omega^{e}}\left(\mathbf{B}^{b}\right)^{T} \mathbf{C}^{b} \mathbf{B}^{b} d \Omega+\int_{\Omega^{e}}\left(\mathbf{B}^{s}\right)^{T} \mathbf{C}^{s} \mathbf{B}^{s} d \Omega
$$

and the load vector by

$$
\mathbf{g}_{i}=\int_{\Omega^{e}} N_{i}\left[\begin{array}{l}
p \\
0 \\
0
\end{array}\right] d \Omega
$$

where

$$
\mathbf{C}^{b}=\frac{E t^{3}}{12\left(1-\nu^{2}\right)}\left[\begin{array}{ccc}
1 & \nu & 0 \\
\nu & 1 & 0 \\
0 & 0 & \frac{1-\nu}{2}
\end{array}\right] \mathbf{C}^{s}=\frac{E t k}{2(1+\nu)}\left[\begin{array}{ll}
1 & 0 \\
0 & 1
\end{array}\right] .
$$

The element stiffness matrix $\mathbf{K}$ is symmetric, positive definite. Using a low-order element as $n p$ is equal to four, the elements are locked in the limitation of thin plate. Therefore, there are various approaches for eliminating locking found in the literatures $[2,3]$. The aim of this paper is to propose a stabilized integration for a quadrilateral plate element such as: (1) apply the curvature smoothing method that is originated with a mesh-free stabilized conforming nodal integration [4] to the first term of Eq. (2.11); (2) approximate the shear strains with independent interpolation functions (namely the MITC4 [5]) into the second term.

By associating the conventional FEM and the CSM developed for mesh-free nodal integration, the presented method for plates is the idea being as follows: (1) elements are present, as in the FEM, but smoothing cells is considered by partitioning element into many sub-cells (2) integration is carried out either on the elements themselves, or over smoothing cells, forming a partition of the elements (3) applying the CSM on each smoothing cell to normalize local curvature and calculate the bending stiffness matrix (4) approximating the shear strains with independent interpolation functions (mixed interpolated tensorial components) proposed by Bathe et al. [5] and then compute the shearing stiffness matrix.

\section{THE CURVATURE SMOOTHING METHOD}

The CSM was proposed by Wang et al. [4] as the normalization of local curvature. A curvature smoothing stabilization is created to compute nodal curvature by a divergence estimation of a spatial averaging of curvature fields. This curvature smoothing avoids evaluating derivatives of mesh-free shape functions at nodes and thus eliminates deflective 
modes. The motivation of this work is to develop the curvature smoothing approach for plate structures in the FEM. A curvature smoothing at an arbitrary point is modified in this paper by

$$
\tilde{\boldsymbol{\kappa}}^{h}\left(\mathbf{x}_{C}\right)=\int_{\Omega^{h}} \kappa^{h}(\mathbf{x}) \Phi\left(\mathbf{x}-\mathbf{x}_{C}\right) d \Omega,
$$

where $\Phi$ is a smoothing function that generally satisfies the following properties $[4,9]$

$$
\Phi \geq 0 \text { and } \int_{\Omega^{h}} \Phi d \Omega=1
$$

For simplicity, $\Phi$ is assumed to be a step function defined by

$$
\Phi\left(\mathbf{x}-\mathbf{x}_{C}\right)=\left\{\begin{array}{l}
1 / A_{C}, \mathbf{x} \in \Omega_{C} \\
0, \mathbf{x} \notin \Omega_{C}
\end{array},\right.
$$

where $A_{C}$ is the area of the smoothing cell, $\Omega_{C} \subset \Omega^{e} \subset \Omega^{h}$, as shown in Fig. 2.



Fig. 2. Example of finite element meshes and smoothing cells

Substituting Eq. (3.3) into Eq. (3.1), and applying the divergence theorem, we obtain

$$
\tilde{\kappa}_{i j}^{h}\left(\mathbf{x}_{C}\right)=\frac{1}{2 A_{C}} \int_{\Omega_{C}}\left(\frac{\partial \theta_{i}^{h}}{\partial x_{j}}+\frac{\partial \theta_{j}^{h}}{\partial x_{i}}\right) d \Omega=\frac{1}{2 A_{C}} \int_{\Gamma_{C}}\left(\theta_{i}^{h} n_{j}+\theta_{j}^{h} n_{i}\right) d \Gamma .
$$

Next, we consider an arbitrary smoothing cell, $\Omega_{C}$ with boundary $\Gamma_{C}=\bigcup_{b=1}^{n b} \Gamma_{C}^{b}$, where $\Gamma_{C}^{b}$ is the boundary segments of $\Omega_{C}$, and $n b$ is the total number of edges of each smoothing cell. The relationship between the smoothed curvature field and the nodal displacement is written

$$
\tilde{\kappa}^{h}=\tilde{\mathbf{B}}^{b} \mathbf{q}
$$


The smoothed element curvature stiffness matrix is obtained by the following form

$$
\tilde{\mathbf{K}}^{b}=\int_{\Omega^{e}}\left(\tilde{\mathbf{B}}^{b}\right)^{T} \mathbf{C}^{b} \tilde{\mathbf{B}}^{b} d \Omega=\sum_{C=1}^{n c}\left(\tilde{\mathbf{B}}^{b}\right)^{T}\left(\mathbf{x}_{C}\right) \mathbf{C}^{b} \tilde{\mathbf{B}}^{b}\left(\mathbf{x}_{C}\right) A_{C}
$$

where $n c$ is number of smoothing cells of the element, see Fig. 3.

Here, the integrands are constant over each $\Omega_{C}$ and the non-local curvature displacement matrix reads

$$
\tilde{B}_{i}^{b}\left(\mathbf{x}_{C}\right)=\frac{1}{A_{C}} \int_{\Gamma_{C}}\left(\begin{array}{ccc}
0 & 0 & N_{i} n_{x} \\
0 & -N_{i} n_{y} & 0 \\
0 & -N_{i} n_{x} & N_{i} n_{y}
\end{array}\right) d \Gamma .
$$

From Eq. (3.7), we can use Gauss points for line integration along each segment of $\Gamma_{C}^{b}$. In approximating bilinear fields, if the shape function is linear on each segment of a cell's boundary, one Gauss point is sufficient for an exact integration.

$$
\tilde{B}_{i}^{b}\left(\mathbf{x}_{C}\right)=\frac{1}{A_{C}} \sum_{b=1}^{n b}\left(\begin{array}{ccc}
0 & 0 & N_{i}\left(\mathbf{x}_{b}^{G}\right) n_{x} \\
0 & -N_{i}\left(\mathbf{x}_{b}^{G}\right) n_{y} & 0 \\
0 & -N_{i}\left(\mathbf{x}_{b}^{G}\right) n_{x} & N_{i}\left(\mathbf{x}_{b}^{G}\right) n_{y}
\end{array}\right) l_{b}^{C},
$$

where $\mathbf{x}_{b}^{G}$ and $l_{b}^{C}$ are the midpoint (Gauss point) and the length of $\Gamma_{b}^{C}$, respectively.

The smoothed curvatures lead to high flexibility such as arbitrary polygonal elements, and a slight computational cost reduction. The element is subdivided into nc non-overlapping sub-domains also called smoothing cells. Fig. 3 is the example of such a division with nc $=$ 1, 2, 3 and 4 corresponding to 1-subcell, 2-subcell, 3-subcell and 4-subcell elements. Then the curvature is smoothed over each sub-cell.

Now we approximate the shear strains with independent interpolation fields in the natural coordinate system. It comes as follows [2]:

$$
\left[\begin{array}{l}
\gamma_{x} \\
\gamma_{y}
\end{array}\right]=\mathbf{J}^{-1}\left[\begin{array}{l}
\dot{\gamma}_{\xi} \\
\gamma_{\eta}
\end{array}\right]
$$

where

$$
\gamma_{\xi}=\frac{1}{2}\left[(1-\eta) \gamma_{\xi}^{B}+(1+\eta) \gamma_{\xi}^{D}\right], \gamma_{\eta}=\frac{1}{2}\left[(1-\xi) \gamma_{\eta}^{A}+(1+\xi) \gamma_{\eta}^{D}\right],
$$

with $\mathbf{J}$ is the Jacobian matrix and the midside nodes A, B, C, D are given in Fig. 1. Presenting $\gamma_{\xi}^{B}, \gamma_{\xi}^{D}$ and $\gamma_{\eta}^{A}, \gamma_{\eta}^{D}$ based on the discretized fields $u^{h}$, we obtain the shear matrix in the following form

$$
\mathbf{B}_{i}^{s}=\mathbf{J}^{-1}\left[\begin{array}{lll}
N_{i, \xi} & -b_{i}^{12} N_{i, \xi} & b_{i}^{11} N_{i, \xi} \\
N_{i, \eta} & -b_{i}^{22} N_{i, \eta} & b_{i}^{21} N_{i, \eta}
\end{array}\right],
$$

where

$$
b_{i}^{11}=\xi_{i} x_{, \xi}^{M}, b_{i}^{12}=\xi_{i} y_{, \xi}^{M}, b_{i}^{21}=\eta_{i} x_{, \eta}^{L}, b_{i}^{22}=\eta_{i} y_{, \eta}^{L},
$$

with $\xi_{i} \in\{-1,1,1,-1\}, \eta_{i} \in\{-1,-1,1,1\}$ and $(i, M, L) \in\{(1, B, A) ;(2, B, C) ;(3, D, C)$; $(4, D, A)\}$

Thus the smoothed element stiffness matrix can be obtained as follows:

$$
\tilde{\mathbf{K}}=\tilde{\mathbf{K}}^{b}+\mathbf{K}^{s}=\sum_{C=1}^{n c}\left(\tilde{\mathbf{B}}^{b}\right)^{T} \mathbf{C}^{b} \tilde{\mathbf{B}}^{b} A_{C}+\int_{\Omega^{e}}\left(\mathbf{B}^{s}\right)^{T} \mathbf{C}^{s} \mathbf{B}^{s} d \Omega,
$$




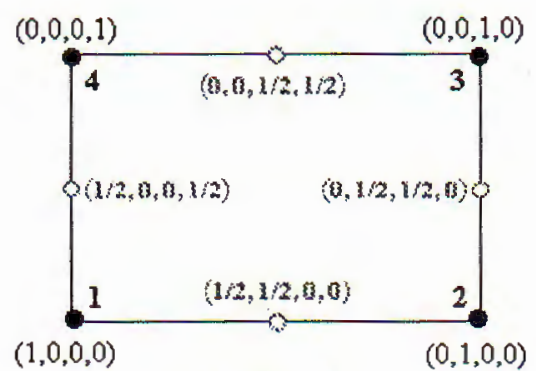

1-Subcell

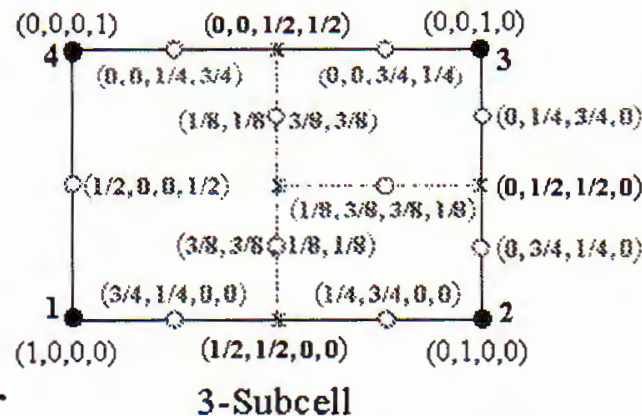

Field node
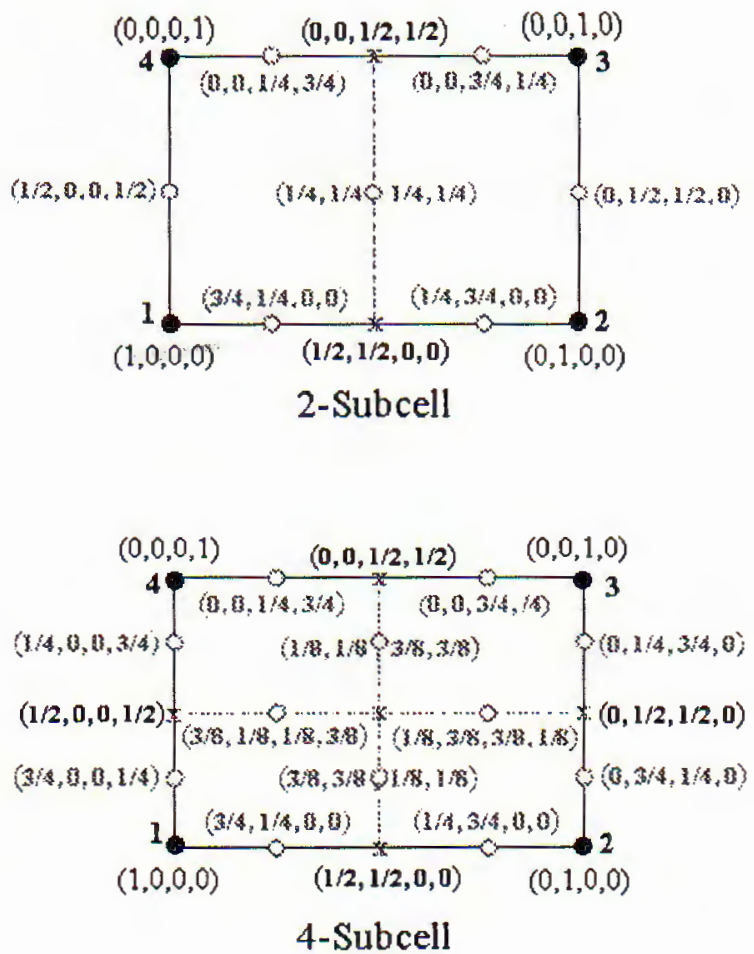

Integration node $\longrightarrow$

Fig. 3. Division of an element into smoothing cells (nc) and the value of the shape function along the boundaries of cells: $k$-Subcell stands for the shape function of the MISCk element, $\mathrm{k}=1,2,3,4$

where the shear term, $\mathbf{K}^{s}$ is still computed by using $2 \times 2$ Gauss points while the element bending stiffness, $\mathbf{K}^{b}$ in Eq. (2.11) is replaced by smoothed curvature technique on each wise-smoothing cell of the element.

\section{NUMERICAL RESULTS}

To coin four-node quadrilateral plate element with stabilized conforming nodal integration, we label to be MISCk (a Mixed Interpolation with Smoothed Curvatures for four-node quadrilateral Reissner-Mindlin plate element) - with smooth $\mathrm{k} \in\{1,2,3,4\}$ subcell on the bending terms. For instance, the MISC1 element is the result of stabilized conforming nodal integration in which only one subcell is used to integrate the bending part of the element stiffness matrix. We also use the reduced/selective integration quadrilateral element (Q4-R) for comparison with the MITC4 and the MISCk elements.

\subsection{Patch test}

First we investigate the element behaviour with the patch test. This is a numerical technique to prove that the proposed method will or will not converge. A plate with five quadrilateral elements is given in Fig. 4. The boundary deflection is assumed to be 
$w=\frac{1}{2}\left(1+x+2 y+x^{2}+x y+y^{2}\right)[10]$. The results shown in Table 1 enjoy that the MITC4 element and the MISCk element pass the patch test while the element Q4-R fails. Also, note that the element $\mathrm{Q} 4$ that uses full numerical integration on both bending and shear terms cannot pass patch test.

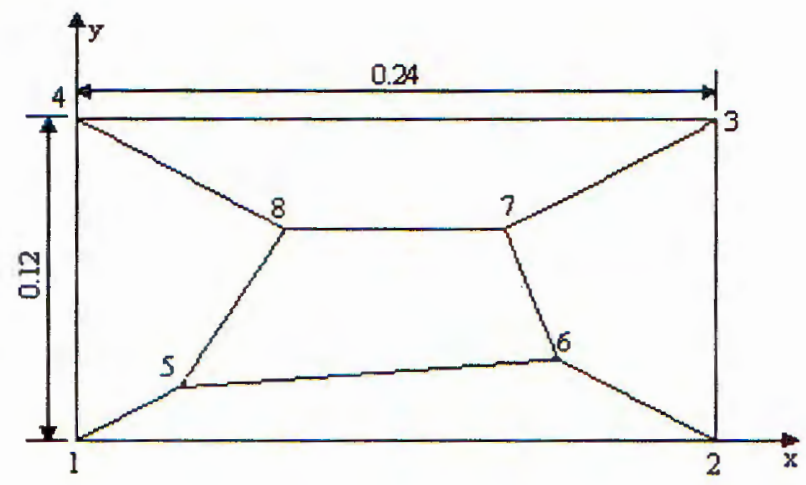

\begin{tabular}{c|cl} 
Node & coordinates \\
1 & 0.0 & 0.0 \\
2 & 0.24 & 0.0 \\
3 & 0.24 & 0.12 \\
4 & 0.0 & 0.12 \\
5 & 0.04 & 0.02 \\
6 & 0.18 & 0.03 \\
7 & 0.16 & 0.08 \\
8 & 0.08 & 0.08
\end{tabular}

Fig. 4. Patch test of elements

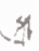

Table 1. Patch test

\begin{tabular}{ccccccc}
\hline Element & $w_{5}$ & $\theta_{x 5}$ & $\theta_{y 5}$ & $m_{x 5}$ & $m_{y 5}$ & $m_{x y 5}$ \\
\hline Q4-R & 0.5440 & 1.0358 & -0.676 & - & - & - \\
MITC4 & 0.5414 & 1.04 & -0.55 & -0.01111 & -0.01111 & -0.00333 \\
MISC1 & 0.5414 & 1.04 & -0.55 & -0.01111 & -0.01111 & -0.00333 \\
MISC2 & 0.5414 & 1.04 & -0.55 & -0.01111 & -0.01111 & -0.00333 \\
MISC3 & 0.5414 & 1.04 & -0.55 & -0.01111 & -0.01111 & -0.00333 \\
MISC4 & 0.5414 & 1.04 & -0.55 & -0.01111 & -0.01111 & -0.00333 \\
Exact & 0.5414 & 1.04 & -0.55 & -0.01111 & -0.01111 & -0.00333 \\
\hline \multicolumn{7}{c}{- no constant moments }
\end{tabular}

\subsection{A simply supported square plate.}

A simply supported square plate is given in Fig. 5 subjected to a uniform load. The geometry and material parameters are given as length $L=10$, thickness $t=0.1$, Young's modulus $E=1092000$, Poisson's ratio $\nu=0.3$ and $p=1$. The series solution considered as analytical solution of Kirchhoff theory is given in [11]. Although a shear correction factor now is chosen to be a large value $k=1000$ that the finite element solutions often converge against the Kirchhoff solution, the convergence behaviour of the MISCk elements in this case is very good for the deflection and the bending moment, see in Fig. 6 .

\subsection{Razzaque's skew plate model}

A rhombic plate with skew angle $60^{\circ}$ simply supported $\operatorname{soft}(w=0)$ on two opposite edges and free on the remainder two edges. This plate which was originally mentioned by Razzaque [12] subjected to a uniform load $p=1$. Problem model and initial mesh with 


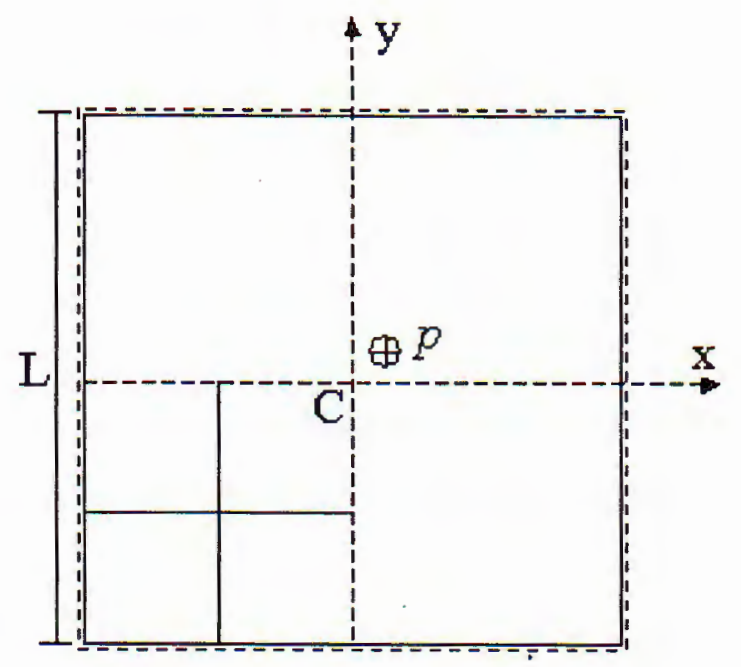

Fig. 5. A simply supported square plate subjected to a uniform load

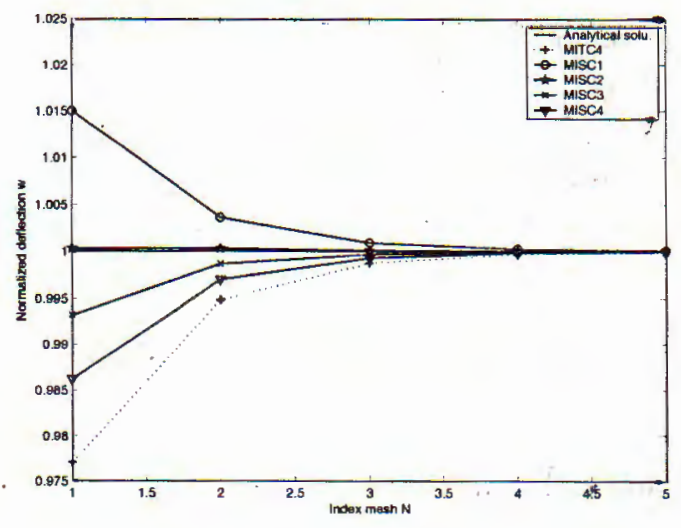

(a)

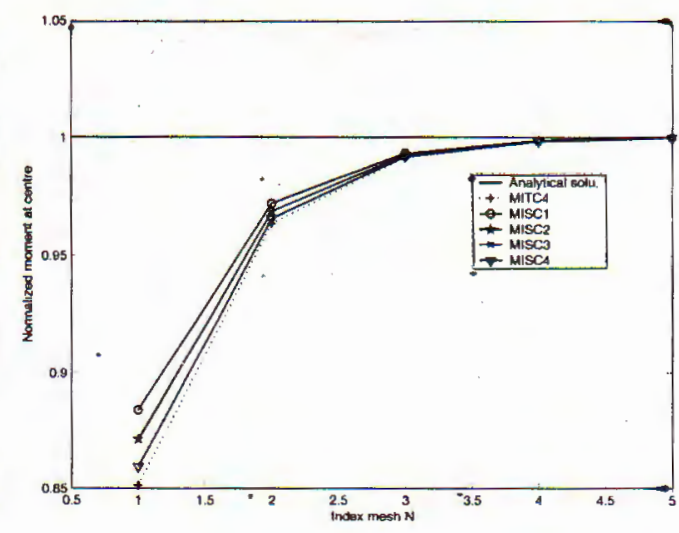

(b)

Fig. 6. Normalized deflection and moment at center of simply support square plate subjected to uniform load

$4 \times 4$ elements are illustrated in Fig. 7a. Data is given as follows: $L=100, t=0.1, E=$ $1092000, \nu=0.3$. The results obtained in Table 2 show that the accuracy of the presented method is always better than that of the MITC4 element.

\section{CONCLUSION}

A quadrilateral plate element based the mixed interpolation with smoothed curvatures has been proposed. Except the MISC1 element that exists two zero energy modes, the MISC2, MISC3 and MISC4 elements maintain a sufficient rank. Moreover all proposed 


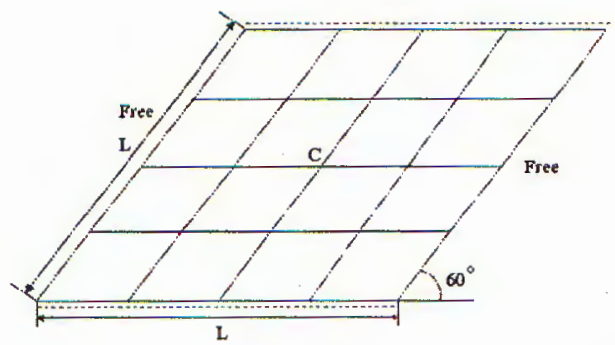

Fig. \%. A simply supported skew plate subjected to a uniform load

Table 2. Central deflection and moment of the Razzaque's skew plate

\begin{tabular}{cccccc}
\hline Mesh & MITC4 & MISC1 & MISC2 & MISC3 & MISC4 \\
\hline & & & & & \\
(a) Central deflection $w_{c} / 10^{4}$ & & & & & \\
$2 \times 2$ & 0.3856 & 0.3648 & 0.3741 & 0.3781 & 0.3816 \\
$4 \times 4$ & 0.6723 & 0.6702 & 0.6725 & 0.6725 & 0.6724 \\
$6 \times 6$ & 0.7357 & 0.7377 & 0.7377 & 0.7370 & 0.7364 \\
$8 \times 8$ & 0.7592 & 0.7615 & 0.7610 & 0.7604 & 0.7598 \\
$12 \times 12$ & 0.7765 & 0.7781 & 0.7776 & 0.7772 & 0.7769 \\
$16 \times 16$ & 0.7827 & 0.7838 & 0.7834 & 0.7832 & 0.7830 \\
$32 \times 32$ & 0.7888 & 0.7892 & 0.7891 & 0.7890 & 0.7889 \\
Reference [12] & & & & & 0.7945 \\
& & & & & \\
(b) Central moment $M_{y} / 10^{3}$ & & & & & \\
$2 \times 2$ & 0.4688 & 0.4688 & 0.4688 & 0.4688 & 0.4688 \\
$4 \times 4$ & 0.8256 & 0.8321 & 0.8301 & 0.8284 & 0.8269 \\
$6 \times 6$ & 0.8976 & 0.9020 & 0.9005 & 0.8994 & 0.8984 \\
$8 \times 8$ & 0.9242 & 0.9272 & 0.9260 & 0.9254 & 0.9245 \\
$12 \times 12$ & 0.9439 & 0.9454 & 0.9448 & 0.9445 & 0.9442 \\
$16 \times 16$ & 0.9510 & 0.9518 & 0.9515 & 0.9513 & 0.9511 \\
$32 \times 32$ & 0.9577 & 0.9580 & 0.9579 & 0.9578 & 0.9578 \\
Reference [12] & & & & & 0.9589 \\
\hline & & & & & \\
\hline
\end{tabular}

elements do not exhibit shear locking in limitation of thin plate. It is also shown that the MISCk element passes patch test. The present method gives a simple computation and gains the slightly high accuracy. Through numerical results we can purify the best element that is the MISC2 element.

Another advantage of the method, emanating from the fact that the bending stiffness matrix is integrated on element boundaries instead of on their interiors is that the proposed formulation still gives accurate and convergent results for extremely distorted meshes.

Our results only derive from computing a uniform refinement of the mesh. Singular problems such as above skew plate or corner plate consume a deal of computational cost with uniform meshes because the convergence is slow. Thereby; a sound knowledge is 


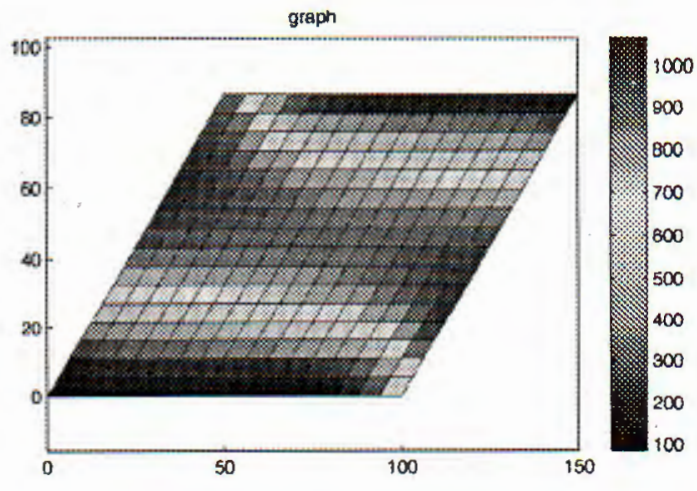

(a)

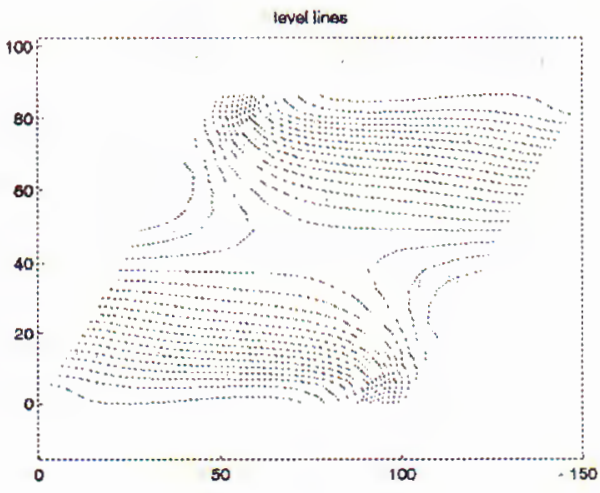

(b)

Fig. 8. A distribution of von Mises and level lines for skew plate using MISC4 element

necessary to generate finite element meshes based on cost-effective and accurate solutions. We thus should combine our method with an adaptive local refinement procedure in order to improve the cost effectiveness of the error bound evaluation.

In addition to the above points, the authors believe that the curvature smoothing technique herein is seamlessly extendable to complex problems such as non-linear material and geometric problems, rid of shear locking in shell formulations.

The above points will be treated in forthcoming papers.

Acknowledgements. The financial support of the Basic Research Project from the Vietnam National Council for Natural Sciences to the authors is greatly acknowledged.

\section{REFERENCES}

1. O. C. Zienkiewicz, R. L. Taylor and J. M. Too, Reduced intgration technique in general analysis of plates and shells Simple and efficient element for plate bending, International Journal for Numerical Methods in Engineering 11(1971) 1529 - 1543.

2. K. J. Bathe, Finite element procedures, Prentice-Hall, Masetchuset(MIT)(1996).

3. O. C. Zienkiewicz and R.L. Taylor, The Finite Element Method, 5th Edition, Butterworth Heinemann(Oxford) (2000).

4. D. Wang and J. S. Chen, Locking - free stabilized conforming nodal integration for meshfree Mindlin-Reissner plate formulation, Computer Methods in Applied Mechanics and Engineering 193(2004) 1065-1083.

5. K. J. Bathe and E. N. Dvorkin, A four-node plate bending element based on Mindlin-Reissener plate theory and a mixed interpolation, International Journal for Numerical Methods in Engineering 21 (1985) $367-383$.

6. G. R. Liu, K. Y. Dai and T. T. Nguyen, A smoothed finite element for mechanics problems, Computational Mechanics(2006) DOI 10.1007/s00466-006-0075-4.

7. X. H. Nguyen, S. Bordas and H. Nguyen Dang, Finite element methods with stabilized conforming nodal integration: convergence, accuracy and properties, International Journal for Numerical Methods in Engineering(2007) in press.

8. J. S. Chen, C. T. Wu, S. Yoon and Y. You, A stabilized conforming nodal integration for Galerkin mesh-free methods, International Journal for Numerical Methods in Engineering 50 (2001) 435-466. 
9. J. W. Yoo, B. Moran and J. S. Chen, Stabilized conforming nodal integration in the naturalelement method, International Journal for Numerical Methods in Engineering 60 (2004) 861890.

10. W. Chen and Y. K. Cheung, Refined quadrilateral element based on Mindlin-Reissner plate theory, International Journal for Numerical Methods in Engineering 47 (2000) 605-627.

11. F. Gruttmann and W. Wagner, A stabilized one-point integrated quadrilateral ReissenerMindlin plate element, International Journal for Numerical Methods in Engineering 61 (2004) $2273-2295$.

12. A. Razzaque, Program for triangular bending elements with derivative smoothing, International Journal for Numerical Methods in Engineering 6 (1973) 333-345.

Received November 15, 2006

\section{ỨNG DỤNG KỸ THUẬT TRƠN HÓA BIẾN DẠNG UỐN CHO PHẦN TỬ TẤM REISSNER-MINDLIN TỨ GIÁC}

Phần tư tứ giác cho tấm Reissner - Mindlin với kỹ thuật trơn hóa biến dạng uốn được đề nghị. Ma trận độ cong tại một điểm bất kỳ được chuẩn hóa bởi hàm làm trơn trong lân cận của điểm khảo sát. Khi hàm trơn được chọn là hăng số, ma trận độ cứng uốn được tính trên biên phần tử thay vì bên trong như cách tính thông thường. Các kết qủa số cho thấy phương pháp đề nghị đạt chính xác cao hơn phương pháp phần tử hữu hạn truyền thống mà không làm tăng chi phí tinh toán. 